\title{
Theoretical investigation of measuring cerebral blood flow in the adult human head using bolus Indocyanine Green injection and near-infrared spectroscopy
}

\author{
Terence S. Leung, Ilias Tachtsidis, Martin Tisdall, Martin Smith, David T. Delpy, and Clare E. Elwell
}

\begin{abstract}
To investigate the accuracy of measuring cerebral blood flow (CBF) using a bolus injection of Indocyanine Green (ICG) detected by near-infrared spectroscopy in adult human heads, simulations were performed using a two-layered model representing the extracerebral and intracerebral layers. Modeled optical data were converted into tissue ICG concentration using either the one-detector modified Beer-Lambert law (MBLL) method, or the two-detector partial path-length (PPL) method. The CBFs were estimated using deconvolution and blood flow index techniques. Using the MBLL method, the CBFs were significantly underestimated but the PPL method improved their accuracy and robustness, especially when used as relative measures. The dispersion of the arterial input function also affected the CBF estimates. (C) 2007 Optical Society of America
\end{abstract}

OCIS codes: $170.3660,170.3890,170.6510$.

\section{Introduction}

The measurement of cerebral blood flow (CBF) is a crucial tool in assessing cerebral well being especially in pathological patients. Regional CBF can be measured with imaging techniques such as dynamic susceptibility contrast magnetic resonance imaging (MRI), xenon computed tomography (CT), and positron emission tomography (PET). These techniques provide a map of CBF for the whole brain but are of limited use to critically ill patients in the intensive care unit who cannot be easily transferred to such scanners. High operating costs also restrict their availability, and for the latter two methods radiation exposure limits the number of studies that can be made. For these reasons, measuring CBF optically at the bedside using nearinfrared spectroscopy (NIRS) is an attractive alterna-

T. S. Leung (tsl@medphys.ucl.ac.uk), I. Tachtsidis, D. T. Delpy, and C. E. Elwell are with the Department of Medical Physics and Bioengineering, University College London, Gower Street, London WC1E 6BT, UK. M. Tisdall and M. Smith are with the Department of Neuroanaesthesia and Neurocritical Care, the National Hospital for Neurology and Neurosurgery, Queen Square, London WC1N 3BG, UK

Received 5 June 2006; revised 6 October 2006; accepted 10 October 2006; posted 12 October 2006 (Doc. ID 71687); published 13 March 2007.

0003-6935/07/101604- $11 \$ 15.00 / 0$

(C) 2007 Optical Society of America tive. The NIRS technique involves placing one or more optical sources and detectors on the surface of the head overlying the cerebral region of interest. Although producing only a single point measurement instead of a 2D cross-sectional map as provided by other imaging modalities, this technique in principle provides a relatively fast, simple, and inexpensive way to measure localized CBF.

While the NIRS measurement of a relative CBF (arbitrary units) can be performed using diffusion correlation spectroscopy, ${ }^{1}$ an absolute CBF (units of $\mathrm{ml} / 100 \mathrm{~g} / \mathrm{min}$ ) has to be measured using tracer tracking techniques. Two main tracers have been used in NIRS, namely, oxy-hemoglobin ${ }^{2}$ and Indocyanine Green (ICG). ${ }^{3,4}$ The use of oxy-hemoglobin as a tracer involves first slightly lowering the subject's inspired oxygen fraction, and then sharply increasing it to produce a bolus of oxy-hemoglobin flowing into the brain, which acts as the tracer. For the first few seconds, the venous outflow of the tracer can be assumed to be zero and the CBF can be calculated from the measurement of changes in arterial oxygen saturation and tissue oxy-hemoglobin concentrations using the Fick principle. The need to reduce oxygen intake can make this technique unsuitable for patients with brain injury, in whom CBF is most likely to be measured. Also since only a few seconds worth of data are used for the CBF calculation, the technique has a large coefficient of variation. ${ }^{2,5}$ 
Another tracer often used is ICG, which is a contrast agent with high absorption in the nearinfrared range, and this produces a signal with a greater signal-to-noise ratio than oxy-hemoglobin. To perform a CBF measurement, a bolus of ICG is injected intravenously, and the arterial ICG concentration may be recorded by a noninvasive pulsedye densitometer with the probe attached to a peripheral site (e.g., the ear lobe, finger, or nose), or by an invasive intra-arterial probe. The tissue ICG concentration is measured by a near-infrared spectrometer with the probe placed on the head overlying the region of interest. There are four main approaches to the calculation of $\mathrm{CBF}$ from the recorded arterial and tissue ICG concentrations. The first approach makes use of only the first few seconds of data when the venous outflow of ICG can be assumed to be zero ${ }^{4}$ and is similar to the method used when oxy-hemoglobin is used as the tracer. The CBF can then be calculated by applying the Fick principle. This approach suffers from the same robustness problem as the oxy-hemoglobin tracer technique because of the relatively small amount of data considered in the calculation.

The second approach considers the slope of the initial rise of the tissue ICG concentration curve, termed the blood flow index (BFI), ${ }^{6-9}$ as an index of CBF. However, BFIs are only comparable when the optical properties of the measurement sites, the geometry, and the circulating blood volume are the same. This is true only during repeated measurements within the same subject. This means that BFIs can be used only as a trend measure to compare $\mathrm{CBF}$ at different times in the same subject with a stable cerebral blood volume (CBV) but not as an absolute measure of CBF among different subjects. Another limitation is that for a valid comparison of different BFI measurements, their arterial input functions (AIFs) have to be exactly the same, which can be difficult to achieve in practice. Zierler ${ }^{10}$ discussed the changes in tissue concentration of a tracer as a function of time and pointed out that the tissue time-concentration curve reflects not only the vascular physiology of the tissue but also the AIF. The BFI is calculated from the initial upslope of the tissue time-concentration curve and does not consider the AIF (the arterial ICG bolus) at all. Using BFIs with different AIFs to compare CBF will therefore introduce significant errors.

The third approach exploits the concept of the impulse residue function (IRF), which carries information about the mean transit time (MTT), CBF, and volume. The IRF is the tracer concentration deposited in tissue when a unit bolus of tracer is injected instantaneously in the arterial site, resembling a delta function. ${ }^{11}$ To recover the IRF, the arterial and tissue ICG concentrations are measured and used for deconvolution. Brown et al. ${ }^{12}$ performed deconvolution with the constraints that the deconvolved IRF was nonnegative and has a flat plateau followed by a decreasing function. The study compared the CBF estimated by using NIRS ICG measurements and using CT measurements in six newborn piglets. Paired $t$-tests showed no significant difference.

The fourth approach was reported by Keller et al. ${ }^{13}$ who derived the venous outflow as the convolution of the arterial inflow and the transport function modeled as a logarithmic normal density function. Using the Fick principle, it was claimed that both the arterial inflow and the venous outflow could be estimated from the measured tissue ICG concentration, and accordingly the MTT could also be found. The CBV was calculated as the division of the tissue ICG concentration by the arterial ICG concentration. Finally, the CBF was derived by dividing the CBV by the MTT. This study also compared the absolute CBFs measured by NIRS and perfusion-weighted MRI in six subjects and found some agreement (the differences against their mean are within two standard deviations).

The NIRS technique may seem attractive, but it poses several challenges when applied to the adult human head. First, its noninvasive nature means that a measurement is taken from the surface of the head and the measurement is subsequently contaminated by an unknown amount of extracerebral blood flow. Second, the AIF is normally measured at a peripheral site and is assumed to mimic the AIF feeding the arteries entering the field of view where the CBF is measured, i.e., the carotid or middle cerebral arteries. As a result, any dispersion of the AIF between the two sites will affect the CBF estimate. These issues have particular implications for CBF measurements in adults with a thick scalp and skull and a more substantial external vasculature.

A study using ICG and NIRS found a mean CBF of $8.3 \mathrm{ml} / 100 \mathrm{~g} / \mathrm{min}$ in 12 healthy adult volunteers using the IRF (deconvolution) method. ${ }^{7}$ This value is very low compared to the $\mathrm{CBF}$ value of approximately $60 \mathrm{ml} / 100 \mathrm{~g} / \mathrm{min}$ as reported by other measurement techniques such as MRI bolus tracking and xenon CT. Indeed, some studies have concluded that measuring CBF with NIRS techniques is unreliable.5,14

Most of the published papers on measuring CBF with NIRS and ICG are experimental studies based on one of the techniques described earlier. To address the two issues raised above, it is necessary to conduct experiments under strictly controlled conditions with known variables, and this is difficult to carry out in practice. We have therefore used physiologically and experimentally informed computer simulations, which are based on an analytical model of light transport in a two-layered medium proposed by Kienle et al..$^{15}$ Both optical attenuation and mean time delay (expressed as a phase delay for a modulated source signal) were calculated.

\section{Methods}

\section{A. Physiological Model}

\section{Indicator-Dilution Theory}

The indicator-dilution theory considers a tracer being injected into the blood stream and mathematically 
relates the blood flow $F$, the $\mathrm{AIF} \mathrm{C}_{\text {art }}(t)$ and the tissue time-concentration curve, $C_{t i s}(t)$ using the concept of the IRF, $R(t)^{16}$ :

$$
C_{t i s}(t)=F \int_{0}^{t} C_{a r t}(\tau) R(t-\tau) \mathrm{d} \tau
$$

where $t$ is time and $\tau$ is an integration variable. The IRF corresponds to the amount of tracer deposited in the tissue, i.e., $C_{t i s}(t)$ when the AIF is a Dirac delta function (its integral is equal to 1 at the time of input and to zero at all other times). The first moment of $R(t)$ is the MTT and the blood volume $(V)$ is $V=$ MTT $\times F$ according to the central volume principle. ${ }^{16}$ Within the context of this study, the AIF corresponds to $[\operatorname{ICG}(t)]_{\mathrm{art}}$, and the tissue time-concentration curves correspond to $[\operatorname{ICG}(t)]_{\mathrm{ext}}$ and $[\operatorname{ICG}(t)]_{\mathrm{int}}$ for the extracerebral layer and the intracerebral layer, respectively. Based on this formulation, we can incorporate known values of blood flow and MTT together with a modeled $[\mathrm{ICG}(t)]_{\text {art }}$, which we simulated based on experimental data, to generate $[\operatorname{ICG}(t)]_{\mathrm{ext}}$ and $[\operatorname{ICG}(t)]_{\mathrm{int}}$.

\section{Physiological Parameters}

Table 1 lists the values of blood flow, blood volume, and MTT used in the simulations. Other parameters in the table will be further discussed in Subsection 2.B.2. Using the xenon CT technique, the blood flow in the extracerebral layer was reported to be 5-8 $\mathrm{ml} / 100 \mathrm{~g} / \mathrm{min} .{ }^{17} \mathrm{~A}$ mean value of $6.5 \mathrm{ml} / 100 \mathrm{~g} / \mathrm{min}$ has been used here. In another study, the mean absolute oxy- and deoxy-hemoglobin concentration in the forehead were found to be 6.4 and $4.3 \mu \mathrm{M}$, respectively using near-infrared spatially resolved spectroscopy with an optical probe having one source and nine detectors (SD spacings ranging from 1.75 to $16.79 \mathrm{~mm}) .{ }^{18}$ The total absolute tissue hemoglobin concentration (i.e., $10.7 \mu \mathrm{M}$ ) was converted to the blood volume of $0.7 \mathrm{ml} / 100 \mathrm{~g}$ for the extracerebral layer in Table 1 . The conversion involves the use of a nominal blood hemoglobin concentration of $14 \mathrm{~g} / \mathrm{dl}$ and a small-to-large-vessel hematocrit ratio of $0.69 .{ }^{19}$ The values for the blood flow and volume in the brain

Table 1. Physiological and Optical Parameters (Baseline Value) ${ }^{a}$

\begin{tabular}{lcc}
\hline & Extracerebral & $\begin{array}{c}\text { Intracerebral } \\
\text { (Gray Matter) }\end{array}$ \\
\hline Blood volume $(\mathrm{ml} / 100 \mathrm{~g})$ & 0.7 & 4 \\
Total Hb concentration, & 11 & 61 \\
$\quad[\mathrm{HbT}](\mu \mathrm{M})$ & & \\
Blood flow $(\mathrm{ml} / 100 \mathrm{~g} / \mathrm{min})$ & $6.5^{+}$ & $60^{+}$ \\
Mean transit time $(\mathrm{s})$ & 6.5 & 4.0 \\
Water content $(\%)$ & 70 & 71 \\
Background absorption & 0.015 & 0.023 \\
$\quad$ coefficient, $\mu_{a}^{b k}\left(\mathrm{~mm}^{-1}\right)$ & & $0^{+}$ \\
Dispersion, $\beta(\mathrm{s})$ & 6 & \\
\hline
\end{tabular}

${ }^{a}$ The superscript ${ }^{+}$denotes the parameter whose value is to be changed in the numerical experiments. were obtained from a MRI CBF simulation study by Calamante et al. ${ }^{20}$ who found them suitable for simulating the blood flow and volume in the gray matter.

\section{Simulation of the Arterial Input Function}

The experimental $[\mathrm{ICG}(t)]_{\mathrm{art}}$ was measured by a pulse dye-densitometer (DDG-2001 Nihon Kohden, Tokyo, Japan) with a nasal probe on a healthy adult volunteer. We found by visual inspection that the lognormal function, which has been used to approximate the arterial input function in the estimation of cardiac output, ${ }^{21}$ also sufficiently fits the first pass of our experimentally measured $[\mathrm{ICG}(t)]_{\mathrm{art}}$. One empirical feature we found in the time-concentration curve of the NIRS measured signal is that the recirculation is more dominant than that measured by other techniques such as MRI and CT. We therefore also consider the recirculation and $[\operatorname{ICG}(t)]_{\mathrm{art}}$ is modeled as the summation of three lognormal functions:

$$
\begin{aligned}
{[\operatorname{ICG}(t)]_{\text {art }} } & =\sum_{n=1}^{3} A_{n} \exp \left[-k_{n} f\left(t, t_{n}\right)^{2}\right], \\
f\left(t, t_{n}\right) & =\begin{array}{cl}
\ln \left[\left(t-\tau_{n}\right) / t_{n}\right] & \text { for } t \geq \tau_{n} \\
0 & \text { otherwise }
\end{array}
\end{aligned}
$$

where $A_{1}=11.5, k_{1}=2.6, t_{1}=4.2 \mathrm{~s}, \tau_{1}=0 \mathrm{~s}, A_{2}=$ $1.1, k_{2}=0.25, t_{2}=25 \mathrm{~s}, \tau_{2}=15 \mathrm{~s}, A_{3}=0.8, k_{3}=4$, $t_{3}=25 \mathrm{~s}$, and $\tau_{3}=1 \mathrm{~s}$. The modeled and experimentally measured $[\operatorname{ICG}(t)]_{\text {art }}$ are plotted in Fig. 1(a) for comparison. Another widely used model for the arterial input function is the gamma variate function. ${ }^{20,22}$

\section{Simulation of the Impulse Residue Functions}

A simple and widely used model for the IRF in tissue is the exponential function, which considers the vasculature bed as one single and well-mixed compartment. It has been used to model the IRF in the brain $^{20,22:} R(t)=\exp (-t / \mathrm{MTT})$ where $t \geq 0$ and the first moment of $R(t)$ is MTT. From the measurement
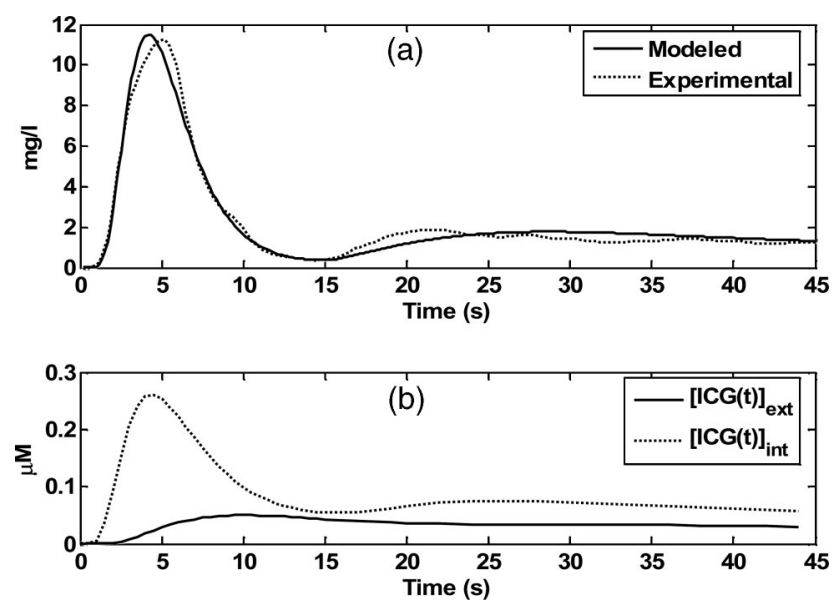

Fig. 1. (a) Modeled and experimentally measured arterial input function $[\operatorname{ICG}(t)]_{\mathrm{art}}$. (b) Modeled tissue ICG concentration time curves in the extracerebral layer $[\operatorname{ICG}(t)]_{\mathrm{ext}}$ and intracerebral layer $[\operatorname{ICG}(t)]_{\text {int }}$. 
site to the site where ICG enters the arteries feeding the tissue in the field of view, $[\mathrm{ICG}(t)]_{\text {art }}$ may be dispersed. The dispersion can be modeled by convolving $R(t)$ with a dispersion function $\exp (-t / \beta) / \beta$ and the effective IRF becomes ${ }^{20}$

$R(t)=\frac{M T T}{(\beta-M T T)}[\exp (-t / \beta)-\exp (-t / M T T)]$,

where $t \geq 0$ and $\beta$ is the dispersion factor in units of seconds.

\section{Simulation of the Tissue Indocyanine Green Concentration Curves}

Using the AIF model, the IRFs, and the MTT and the blood flow values shown in Table $1,[\operatorname{ICG}(t)]_{\text {ext }}$ and $[\operatorname{ICG}(t)]_{\text {int }}$ can be simulated as shown in Fig. 1(b). It can be seen that $[\operatorname{ICG}(t)]_{\mathrm{ext}}$ is more dispersed and has a lower peak value, which agrees with the general behavior of the tissue tracer time-concentration curve in the extracerebral layer found in a MRI study. ${ }^{23}$

\section{B. Optical Model}

\section{Two-Layered Analytical Model}

To simulate the light propagation in the extracerebral layer and the intracerebral layer, a two-layered model for frequency domain measurements proposed by Kienle et $a l .{ }^{15}$ has been adopted. Given the depth of the first layer, the modulation frequency $\left(f_{m o d}\right)$, the absorption coefficients $\left(\mu_{a}\right)$ and the reduced scattering coefficients $\left(\mu_{s}^{\prime}\right)$ in the upper and lower layers, this model can predict the reflectance $(r)$ at a predefined SD spacing and the phase angle $(\theta)$ between the source and the detector. The model assumes a flat geometry both at the surface and at the boundary between the upper and the lower layers, and the lower layer is infinitely thick. It also assumes a tissue-air refractive index mismatch.

\section{Optical Parameters}

Using the two-layered model, we can predict both the reflectance and the phase angles at different surface measurement points with assigned $\mu_{a}$ and $\mu_{s}^{\prime}$ in the two layers. Here the two optical coefficients are expressed as $\mu_{a}(\lambda)$ and $\mu_{s}^{\prime}(\lambda)$ to highlight their wavelength dependency. Two equations have been used to form $\mu_{a}(\lambda)$ in each layer. The first equation calculates the $\mu_{a}(\lambda)$ before the ICG enters the field of view and is denoted by $\mu_{a}^{0}(\lambda)$ here:

$$
\begin{aligned}
\mu_{a}^{0}(\lambda)= & \varepsilon_{H H b}(\lambda)[H b T]\left(1-S O_{2}\right)+\varepsilon_{H b O_{2}}(\lambda)[H b T] S O_{2} \\
& +\mu_{a, H_{2} O}(\lambda) W+\mu_{a}^{b k},
\end{aligned}
$$

where $\varepsilon_{H H b}(\lambda)$ and $\varepsilon_{H b O_{2}}(\lambda)$ are the specific absorption coefficients of deoxy- and oxy-hemoglobin $\left(\mu \mathrm{M}^{-1} \mathrm{~mm}^{-1}\right)$, $[H b T]$ is the total hemoglobin concentration $(\mu \mathrm{M})$, $\mathrm{SO}_{2}$ is the tissue oxygenation (as a fraction), $W$ is the water content (as a fraction), and $\mu_{a, \mathrm{H}_{2} \mathrm{O}}(\lambda)$ is the absorption coefficient of $100 \%$ water $\left(\mathrm{mm}^{-1}\right)$, and $\mu_{a}^{b k}$ is a wavelength independent background absorption $\left(\mathrm{mm}^{-1}\right)$. It is assumed that the physiology does not change over time, and subsequently these parameters are assumed to be time invariant. The values of $[H b T]$ and $W$ for each layer are listed in Table 1 . The water content of each layer has been measured previously using the MRI technique. ${ }^{24}$ The $\mathrm{SO}_{2}$ for the two layers has been set to a nominal value of 0.7 . The $\mu_{a}(\lambda)$ in the two layers calculated using these parameters was smaller than that used by Okada and Delpy. ${ }^{25} \mathrm{~A}$ wavelength-independent background $\mu_{a}^{b k}$ was therefore added so that the total value matched those in Okada and Delpy ${ }^{25}$ at $800 \mathrm{~nm}$, i.e., the $\mu_{a}^{b k}$ for the extracerebral layer $=0.015 \mathrm{~mm}^{-1}$ and the $\mu_{a}^{b k}$ for the intracerebral layer $=0.023 \mathrm{~mm}^{-1}$. The existence of the background absorption was discussed previously by Essenpreis et al. ${ }^{26}$ and Hueber et al. ${ }^{27}$ Using these values, the total extracerebral $\mu_{a}(800 \mathrm{~nm})=$ $0.018 \mathrm{~mm}^{-1}$, and the total intracerebral $\mu_{a}(800 \mathrm{~nm})=$ $0.036 \mathrm{~mm}^{-1}$, which were the same values used in Okada and Delpy. ${ }^{25}$ For comparison this approach also resulted in extracerebral and intracerebral $\mu_{a}(759 \mathrm{~nm})$ of $0.019 \mathrm{~mm}^{-1}$ and $0.037 \mathrm{~mm}^{-1}$, respectively, which are comparable to the experimentally found extracerebral and intracerebral $\mu_{a}(759 \mathrm{~nm})$ of 0.015 and $0.030 \mathrm{~mm}^{-1}$ found in a previous study. ${ }^{28}$

When ICG enters the field of view, a time-variant absorption change is introduced and the corresponding $\mu_{a}$, i.e., $\mu_{a}^{I C G}(t, \lambda)$ can be modeled as

$$
\mu_{a}^{I C G}(t, \lambda)=\mu_{a}^{0}(\lambda)+[I C G(t)] \varepsilon_{I C G}(\lambda),
$$

where $\varepsilon_{I C G}(\lambda)$ is the specific absorption coefficient of ICG. The $[\operatorname{ICG}(t)]$ here corresponds to either $[\operatorname{ICG}(t)]_{\text {ext }}$ or $[\operatorname{ICG}(t)]_{\text {int }}$ whose generation has been discussed in Subsection 2.A. The values of $\varepsilon_{H н b}(\lambda)$, $\varepsilon_{\mathrm{HbO} 2}(\lambda), \varepsilon_{I C G}(\lambda)$, and $\mu_{a, \mathrm{H} 2 \mathrm{O}}(\lambda)$ used here were obtained from previous measurements performed in our laboratory. ${ }^{4,29}$ It should be noted that all the absorption coefficients here are based on the logarithm base of $e$, rather than 10 , in order to be consistent with the diffusion theory.

For the $\mu_{s}^{\prime}(\lambda)$ in the extracerebral layer, we employed an expression derived by Doornbos et al. ${ }^{18}$ who conducted spatially resolved spectroscopy measurement on the human forehead: $\mu_{s}^{\text {'ext }}(\lambda)=a\left(\lambda \times 10^{-3}\right)^{-b}$ $\times 10^{-3}$ where $a=1141, b=0.84$, and $\lambda$ is in nanometers. In this study, the maximum SD spacing is $16.79 \mathrm{~mm}$, and the results are applicable mainly to the extracerebral layer.

van der Zee et al. ${ }^{30}$ measured $\mu_{s}^{\prime}$ for brain tissues in vitro at $800 \mathrm{~nm}$. Matcher et al. ${ }^{31}$ measured $\mu_{s}^{\prime}(\lambda)$ in the adult human forehead in vivo using time-resolved spectroscopy with a SD spacing of $40 \mathrm{~mm}$, which was believed to probe both the extracerebral layer and the intracerebral layer. Here we used $\mu_{s}^{\prime}(800 \mathrm{~nm})=2.2$ $\mathrm{mm}^{-1}$ for the gray matter found by van der Zee et al. ${ }^{30}$ as the reference value and adopted the wavelength dependency found by Matcher et al. ${ }^{31}$ to calculate $\mu_{s}^{\prime}(\lambda)$ at other wavelengths. The formulation of $\mu_{s}^{\prime}(\lambda)$ in the intracerebral layer is written as: $\mu_{s}^{\text {int }}(\lambda)=$ $2.2(\lambda a+b) /(800 a+b)$ where $a=-6.5 \times 10^{-4}$, 
$b=1.45$, and $\lambda$ is in nanometers. For $\lambda=800 \mathrm{~nm}$, $\mu_{s}^{\text {int }}(\lambda)=2.2 \mathrm{~mm}^{-1}$.

Using the techniques discussed in this subsection, the reflectance and phase angles were predicted at 775,813 , and $853 \mathrm{~nm}$, which were three of the wavelengths used in the spectrometer during a previous in vivo study. ${ }^{7}$ The attenuations were calculated from reflectance, i.e., $A=-\log _{e}(r)$, and the total mean path length calculated from the phase angles, i.e., $L=$ $\theta /\left(2 \pi f_{\text {mod }} c_{t i s}\right)$, where $c_{t i s}$ is the speed of light in tissue. The optical data were predicted at two measurement points with SD spacings of 45 and $50 \mathrm{~mm}$, which were chosen to be the same as the configuration of the optodes used in a previous study. ${ }^{7}$

Before ICG entered the field of view, the path length at a SD spacing of $50 \mathrm{~mm}$ is $310 \mathrm{~mm}$ at $813 \mathrm{~nm}$, which corresponds approximately to a differential path-length factor (DPF) of 6.2. This value agrees reasonably well with the experimental finding of 6.1 at $807 \mathrm{~nm}$, using the age-dependent equation of $\mathrm{DPF}=4.99+0.067 \mathrm{~A}^{0.814}$ where A (age) $=30$ years, ${ }^{32}$ and adds to our confidence that the two-layered analytical model and the optical and physiological parameters used (as shown in Table 1) are suitable.

\section{Conversion of Optical Data to Concentrations}

\section{Modified Beer-Lambert Law Method}

One widely used technique to convert attenuation into ICG concentration is by using the MBLL ${ }^{4,7,12}$ : $\Delta \mathbf{C}=\boldsymbol{\varepsilon}^{-1} \Delta \mathbf{A} / \mathrm{L}$ where $L$ is the optical mean path length, $\Delta \mathbf{C}$ is a column vector containing the changes in concentrations of ICG, deoxy- $(\mathrm{HHb})$ and oxyhemoglobin $\left(\mathrm{HbO}_{2}\right)$, i.e., $\left[\Delta C_{I C G} \Delta C_{H H b} \Delta C_{H b O_{2}}\right]^{\mathrm{T}}, \varepsilon^{-1}$ is the inverse of a matrix containing the specific absorption coefficients of ICG, $\mathrm{HHb}$, and $\mathrm{HbO}_{2}$ at different wavelengths, and $\mathbf{A}$ is a column vector containing changes in attenuation at different wavelengths. In this work, $\Delta \mathbf{A}=\left[A^{\mathrm{ICG}}\left(\lambda_{1}\right)-A^{0}\left(\lambda_{1}\right), A^{I C G}\left(\lambda_{2}\right)-A^{0}\left(\lambda_{2}\right)\right.$, $\left.A^{I C G}\left(\lambda_{3}\right)-A^{0}\left(\lambda_{3}\right)\right]^{\mathrm{T}}=\left[\Delta A\left(\lambda_{1}\right), \Delta A\left(\lambda_{2}\right), \Delta A\left(\lambda_{3}\right)\right]^{\mathrm{T}}$ where $A^{0}(\lambda)$, and $A^{I C G}(\lambda)$ are attenuations before and after ICG enters the field of view, and the three wavelengths used are 775, 813, and $853 \mathrm{~nm}$. Since the concentrations of $\mathrm{HHb}$ and $\mathrm{HbO}_{2}$ are set to be constant before and after the appearance of ICG, it is expected that the resulting $\Delta C_{\mathrm{HHb}}$ and $\Delta C_{\mathrm{HbO}_{2}}$ are close to zero. It should be noted that $L$ is in fact wavelength dependent but a fixed value is often used and scaling factors correcting for the wavelength dependency are incorporated in $\varepsilon$. This formulation assumes a homogeneous medium. We refer to the use of a single-detector measurement and the MBLL as the MBLL method.

\section{Partial Path-Length Method}

Hiraoka et al. ${ }^{33}$ introduced the concept of partial path lengths to extend the MBLL to heterogeneous media. The partial path length of a layer is defined as a partial derivative of the measured $A$ versus the $\mu_{a}$ at that layer and can be considered as the mean path length that light has traveled in that layer. For a two-layered model with two detectors, we have

$$
\left[\begin{array}{l}
\Delta A_{1}(\lambda) \\
\Delta A_{2}(\lambda)
\end{array}\right]=\left[\begin{array}{ll}
\rho_{1,1}(\lambda) & \rho_{1,2}(\lambda) \\
\rho_{2,1}(\lambda) & \rho_{2,2}(\lambda)
\end{array}\right]\left[\begin{array}{l}
\Delta \mu_{a, 1}(\lambda) \\
\Delta \mu_{a, 2}(\lambda)
\end{array}\right]
$$

where $\Delta A_{1}(\lambda)$ and $\Delta A_{2}(\lambda)$ are the changes of attenuation [i.e., $A^{I C G}(\lambda)-A^{0}(\lambda)$ ] measured by detectors 1 and $2, \Delta \mu_{a, 1}(\lambda)$ and $\Delta \mu_{a, 2}(\lambda)$ are the changes in the absorption coefficient in layers 1 (upper) and 2 (lower), $\rho_{m, n}(\lambda)$ is the partial path length at layer $n$ as measured by detector $m$ ( $m, n=1$ or 2). Subsequently, $\Delta \mu_{a}(\lambda)$ in both layers can be estimated by $\Delta \mathbf{M}=\mathbf{R}^{-1} \Delta \mathbf{A}$, where $\Delta \mathbf{M}=\left[\Delta \mu_{a, 1}(\lambda), \Delta \mu_{a, 2}(\lambda)\right]^{\mathrm{T}}$ and $\mathbf{R}$ is the matrix containing all the partial path lengths $\rho_{m, n}(\lambda)$ in Eq. (6). The partial path lengths, however, cannot be measured directly but can be estimated iteratively with Monte Carlo simulations or a solution of the diffusion equation using frequency or time domain measurements. ${ }^{28}$ In this work they are estimated by $\rho_{1,1}(\lambda)=\Delta A_{1}(\lambda) / \Delta \mu_{a, 1}(\lambda)$, where $\Delta \mu_{a, 1}(\lambda)$ is set to a small value, e.g., 0.001 , and $\Delta A_{1}(\lambda)$ is the corresponding change of attenuation predicted by the optical model, and subsequently, $\rho_{1,2}(\lambda)=L_{1}(\lambda)$ $-\rho_{1,1}(\lambda)$. The corresponding physiological and optical parameters are the baseline values as shown in Table 1 . Both $\rho_{2,1}(\lambda)$ and $\rho_{2,2}(\lambda)$ can be found similarly. Once the $\Delta \mu_{a}(\lambda)$ in the brain, i.e., $\Delta \mu_{a, 2}(\lambda)$, becomes available, the corresponding concentrations can be calculated by $\Delta \mathbf{C}=\varepsilon^{-1} \Delta \mu_{a, 2}$. Here we refer to the use of two detector measurements and the partial path-length formulation as the PPL method. As an example, using the modeling techniques described here and the parameters in Table 1, the partial path length in the brain and the mean path length were estimated to be 18.8 and $254.5 \mathrm{~mm}$ for $\lambda=775 \mathrm{~nm}$, SD spacing $=$ $45 \mathrm{~mm}$ and the thickness of the first layer $=8 \mathrm{~mm}$. The ratio of partial to mean path length is 0.069 . For comparison a previous study 28 in which a timeresolved spectrometer and Monte Carlo simulations were used found the partial path length in the brain and the mean path length of an adult subject to be 26.4 and $206.6 \mathrm{~mm}$ for $\lambda=759 \mathrm{~nm}$, SD spacing $=$ $30 \mathrm{~mm}$ and the thickness of the scalp and skull = $7.9 \mathrm{~mm}$. The corresponding ratio of partial to mean path length is 0.128 . The discrepancies between these results can be attributed to different assumptions and optical properties used.

Using either the MBLL method or the PPL method, the tissue ICG concentration can be calculated. When the MBLL method is used, the notation $[\operatorname{ICG}(t)]_{\operatorname{det} 2}$ is used to denote the tissue ICG concentration as measured by detector $2(\mathrm{SD}$ spacing $=50 \mathrm{~mm})$. When the PPL method is used, the notation $[\operatorname{ICG}(t)]_{\operatorname{det} 1 \& 2}$ is used to indicate that both detectors 1 (SD spacing = $45 \mathrm{~mm})$ and 2 are used. Figure 2 depicts $[\operatorname{ICG}(t)]_{\operatorname{det} 2}$ and $[\operatorname{ICG}(t)]_{\operatorname{det} 1 \& 2}$ predicted with three different dispersion factors in the intracerebral layer (i.e., $\beta=0$, 0.5 , and $1 \mathrm{~s}$ ) and an extracerebral thickness of $10 \mathrm{~mm}$. It should be noted that $[\operatorname{ICG}(t)]_{\operatorname{det} 2}$ accounts for a weighted average of the tissue ICG concentrations in the extracerebral layer, i.e., $[\operatorname{ICG}(t)]_{\text {ext }}$ and the intracerebral layer, $[\operatorname{ICG}(t)]_{\text {int }}$ because of the assumption of a homogeneous medium. On the other 

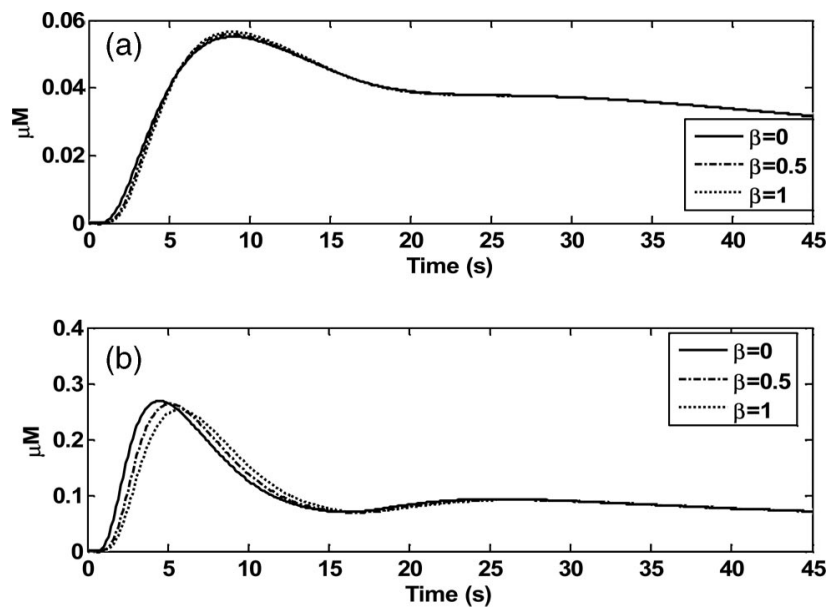

Fig. 2. (a) Tissue ICG time-concentration curves calculated by the MBLL method using detector 2 (SD spacing $=50 \mathrm{~mm}),[\mathrm{ICG}(t)]_{\operatorname{det} 2}$ with three different dispersion factors, $\beta=0,0.5$, and $1 \mathrm{~s}$. (b) Tissue ICG time-concentration curves calculated by the PPL method using detectors 1 and 2 (SD spacings $=45$ and $50 \mathrm{~mm}$ ), $[\operatorname{ICG}(t)]_{\operatorname{det} 1 \& 2}$ with three different dispersion factors, $\beta=0,0.5$, and $1 \mathrm{~s}$.

hand, $[\operatorname{ICG}(t)]_{\operatorname{det} 1 \& 2}$ corresponds to $[\operatorname{ICG}(t)]_{\text {int }}$ only because of the two-layer assumption. It will be shown in Section 3 that the PPL method will provide CBF estimates less sensitive to the extracerebral blood flow. It can be seen from Fig. 2(a) that $[\operatorname{ICG}(t)]_{\operatorname{det} 2}$ is more dispersed and has a lower peak value, bearing more similarities to $[\mathrm{ICG}(t)]_{\mathrm{ext}}$ than $[\mathrm{ICG}(t)]_{\mathrm{int}}$ [see Fig. 1(a)]. This indicates the influence of the extracerebal blood flow on $[\operatorname{ICG}(t)]_{\operatorname{det} 2}$. On the other hand, $[\operatorname{ICG}(t)]_{\operatorname{det} 1 \& 2}$ in Fig. 2(b) is more similar qualitatively to $[\operatorname{ICG}(t)]_{\text {int }}$ showing a high sensitivity to intracerebral changes.

\section{Estimation of Cerebral Blood Flow}

\section{Deconvolution}

Rewriting Eq. (1) in discrete form for the tracer ICG:

$$
\left[\operatorname{ICG}\left(t_{j}\right)\right]_{t i s}=\Delta t \cdot F \cdot \sum_{i=0}^{j}\left[\operatorname{ICG}\left(t_{j}\right)\right]_{\text {art }} \cdot R\left(t_{j}-t_{i}\right),
$$

where $[\operatorname{ICG}(t)]_{\mathrm{tis}}$ is the tissue ICG concentration measured by either the MBLL method or the PPL method as discussed in the previous subsection, and $\Delta t$ is the sampling interval. Deconvolution is basically a numerical process to recover $F \cdot R(t)$ from the measurements $[\mathrm{ICG}(t)]_{\text {art }}$ and $[\mathrm{ICG}(t)]_{\text {tis. }}$ The singular value decomposition (SVD) approach has been used to perform deconvolution in general ${ }^{34}$ and in CBF measurements with MRI bolus tracking. ${ }^{35}$ The SVD has a threshold that allows it to handle noisy data (a higher threshold for a higher level of noise) and maintain a stable solution. Since $R(t)$ has a maximum value of 1 , the maximum value of $\mathbf{x}=\left[F \cdot R\left(t_{0}\right)\right.$, $\left.F \cdot R\left(t_{1}\right), \ldots, F \cdot R\left(t_{N-1}\right)\right]^{T}$ is $F$, i.e., CBF. In actual measurements, there is a time delay between $[\mathrm{ICG}(t)]_{\text {art }}$ measured at a peripheral site and $[\operatorname{ICG}(t)]_{\text {tis }}$ measured over the head, potentially lead- ing to erroneous results after deconvolution. However, the time delay can be compensated for by manually synchronizing the rise of the two curves.

\section{Blood Flow Index}

As an alternative to deconvolution, relative CBF can be quantified using the BFI, ${ }^{6}$ which is defined here as the gradient of $[\mathrm{ICG}(t)]_{\mathrm{tis}}$ during the initial inflow of ICG, i.e., the difference between $90 \%$ and $10 \%$ of the peak value over the corresponding time interval. ${ }^{7}$

\section{E. Numerical Experiments}

Three numerical experiments were performed to investigate different aspects of the $\mathrm{CBF}$ and $\mathrm{BFI}$ estimations:

Numerical Experiment 1: The extracerebral blood flow was fixed at $6.5 \mathrm{ml} / 100 \mathrm{~g} / \mathrm{min}$, while the CBF varied between $-20 \%$ and $+20 \%$ (in steps of $10 \%$ ) around the baseline value of $60 \mathrm{ml} / 100 \mathrm{~g} / \mathrm{min}$.

Numerical Experiment 2: The extracerebral blood flow varied between $-20 \%$ and $+20 \%$ (in steps of $10 \%$ ) around the baseline value of $6.5 \mathrm{ml} / 100 \mathrm{~g} / \mathrm{min}$, while the $\mathrm{CBF}$ was fixed at $60 \mathrm{ml} / 100 \mathrm{~g} / \mathrm{min}$.

Numerical Experiment 3: Both the extracerebral blood flow and $\mathrm{CBF}$ were fixed at 6.5 and 60 $\mathrm{ml} / 100 \mathrm{~g} / \mathrm{min}$, while the dispersion factor in the vasculature varied between 0 and $1 \mathrm{~s}$ (in steps of $0.25 \mathrm{~s}$ ).

The dispersion factor in the vasculature was set to zero for Numerical Experiments 1 and 2. Table 2 summarizes the values of all the parameters used in the three experiments. The values of other parameters such as the water content, background absorption, and reduced scattering coefficients were the same as those shown in Table 1. Kohl-Bareis et al. ${ }^{23}$ found that the MRI perfusion signals using gadolinium-DTPA as a tracer were more dispersed in shape at the extracerebral site than those at the intracerebral site. To incorporate this finding, a larger dispersion factor of $6 \mathrm{~s}$ was used for $R(t)$ in the extracerebral layer. Also, $R(t)$ was normalized to give a maximum value of 1 so that the maximum value of the deconvolved $F \cdot R(t)$ corresponds to the extracerebral blood flow. In each numerical experiment, the optical data (attenuation and phase angle) were predicted from the two-layered analytical model while the thickness of the upper layer varied between 8 and $12 \mathrm{~mm}$ in steps of $2 \mathrm{~mm}$.

Table 2. Parameters Used in the Three Numerical Experiments

\begin{tabular}{cccc}
\hline Numerical & $\begin{array}{c}\text { Extracerebral } \\
\text { Blood Flow } \\
\text { Experiment } \\
(\mathrm{ml} / 100 \mathrm{~g} / \mathrm{min})\end{array}$ & $\begin{array}{c}\text { Cerebral } \\
\text { Blood Flow } \\
(\mathrm{ml} / 100 \mathrm{~g} / \mathrm{min})\end{array}$ & $\begin{array}{c}\text { Dispersion Factor } \\
\text { in the } \\
\text { Intracerebral } \\
\text { Layer } \beta(\mathrm{s})\end{array}$ \\
\hline 1 & 6.5 & $48,54,60$, & 0 \\
2 & $5.2,5.85,6.5$, & 66,72 & 0 \\
& $7.15,7.8$ & 60 & $0,0.25,0.5$, \\
3 & 6.5 & 60 & $0.75,1$ \\
\hline
\end{tabular}


In each numerical experiment, the predicted optical data were analyzed by the MBLL method and the PPL method. When using the MBLL, the mean path length needs to be calculated from the phase angle. Strictly speaking, the mean path length $L(t, \lambda)$ is both time and wavelength dependent. In reality, the experimentally measured phase angles are often very noisy, which in turn affects the accuracy of the estimated $L(t, \lambda)$. Therefore one average phase angle is often used for a period of time. In this work we also used one phase angle (the point before the ICG appears) at each wavelength generated by the twolayered analytical model to calculate one $L(\lambda)$ for the entire tissue ICG time-concentration curve. To use the PPL method, the partial path lengths in the upper and lower layers as measured by the two detectors, i.e., $\rho_{m, n}(m, n=1$ or 2$)$ were estimated using a first-order approximation as described in Subsection 2.C.2. The partial path lengths are also time and wavelength dependent, i.e., $\rho_{m, n}(t, \lambda)$, but to estimate them accurately using experimental measurements we need to know the evolution of $\mu_{a}(t, \lambda)$ in the two layers, which is in fact what we are trying to measure. Therefore it was assumed that the partial path lengths did not change significantly during the inflow and clearance of the ICG, and they were estimated before the ICG appeared in the field of view. This assumption inevitably introduced certain errors, which we will further discuss later. For both methods, the threshold in the deconvolution was set to a rather small value of $1 \%$ of the maximum singular value because no noise was present in the predicted data.

\section{Results}

Here we report the results obtained from the three numerical experiments. For each experiment, the estimated CBFs are given in both absolute units $(\mathrm{ml} / 100 \mathrm{~g} / \mathrm{min})$ and relative units (\% change). The estimated absolute CBFs are biased to different degrees owing to the presence of the extracerebral blood flow in all cases. However, the estimated relative percentage changes in CBFs are in some cases quite accurate. For Numerical Experiments 1 and 2 (Figs. 3 and 4), all the relative percentage changes were calculated with respect to the middle value (out of five values), while in Numerical Experiment 3 (Fig. 5) the results corresponding to the dispersion factor $\beta=$ $0 \mathrm{~s}$ (the first value) were used as the reference values.

The absolute BFI values in terms of $\mu \mathrm{M} / \mathrm{s}$ depend on the optical properties of the measurement site and the total circulating blood volume, which are likely to differ in different subjects. Therefore absolute BFIs cannot be compared between different subjects. Using BFI in the same subject at different times, relative percentage changes in BFIs are sufficient. Therefore we present only the results of relative percentage changes in BFIs here but not the absolute values.

Figures 3, 4, and 5 show the results of the three numerical experiments. In all the figures, the three panels on the left are the results obtained using the
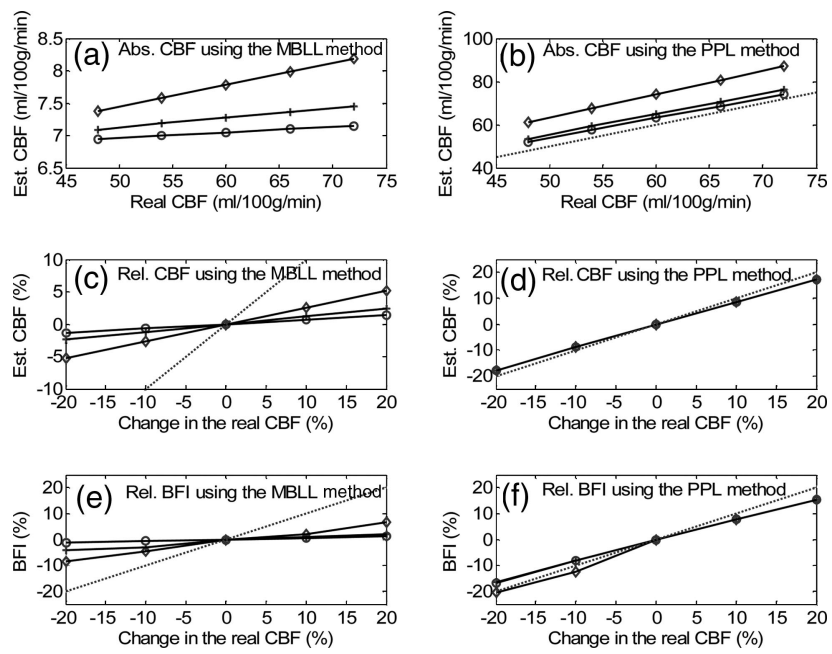

Fig. 3. Numerical Experiment 1: Extracerebral blood flow (the upper layer) was kept constant at $6.5 \mathrm{ml} / 100 \mathrm{~g} / \mathrm{min}$, and the real CBF (the lower layer) varied between $-20 \%$ and $20 \%$ of its baseline value at $60 \mathrm{ml} / 100 \mathrm{~g} / \mathrm{min}$. (a), (c), and (e) Results obtained using the MBLL method; (b), (d), and (f) results obtained using the PPL method. The results are presented as absolute values $(\mathrm{ml} / 100$ $\mathrm{g} / \mathrm{min}$ ) for estimated CBFs in (a) and (b), and as relative values (\% change from the middle value) for estimated CBFs and BFIs in (c), (d), (e), and (f). Different thicknesses of the extracerebral layer at 8 $(\diamond), 10(+)$ and $12(\bigcirc) \mathrm{mm}$ were used. The dotted line represents the ideal estimation of $\mathrm{CBF}$, which follows exactly the real CBF.

MBLL method (single detector with $50 \mathrm{~mm}$ SD spacing) while the three on the right are the results obtained using the PPL method (two detectors with
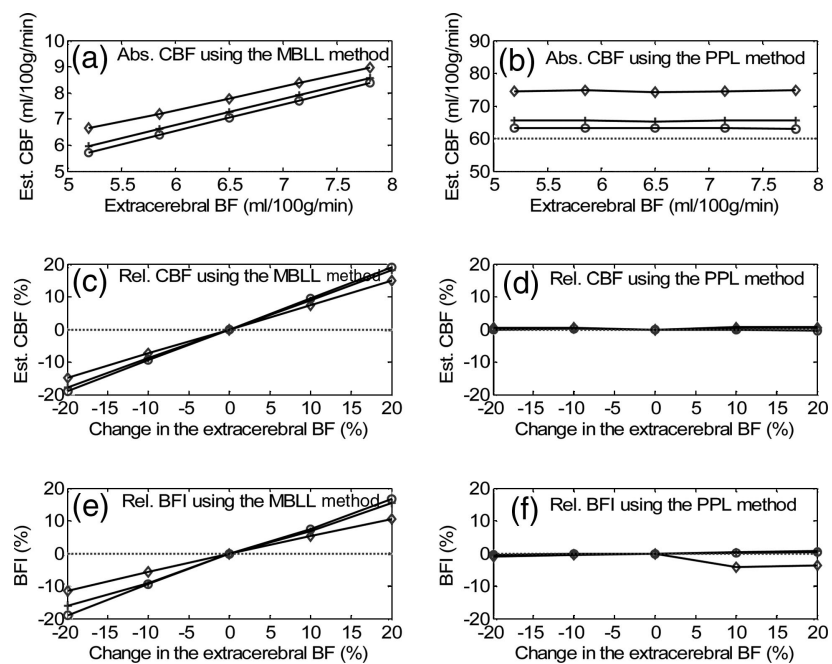

Fig. 4. Numerical Experiment 2: CBF (the lower layer) was kept constant at $60 \mathrm{ml} / 100 \mathrm{~g} / \mathrm{min}$, and the extracerebral blood flow (the upper layer) varied between $-20 \%$ and $20 \%$ of its nominal value at $6.5 \mathrm{ml} / 100 \mathrm{~g} / \mathrm{min}$. (a), (c), and (e) Results obtained using the MBLL method. (b), (d), and (f) Results obtained using the PPL method. The results are presented as absolute values $(\mathrm{ml} / 100 \mathrm{~g} / \mathrm{min})$ for estimated CBFs in (a) and (b) and as relative values (\% change from the middle value) for estimated CBFs and BFIs in (c), (d), (e), and (f). Different thicknesses of the extracerebral layer at $8(\diamond), 10(+)$ and $12(\bigcirc) \mathrm{mm}$ were used. The dotted line represents the ideal estimation of $\mathrm{CBF}$, which is not biased by the extracerebral blood flow. 

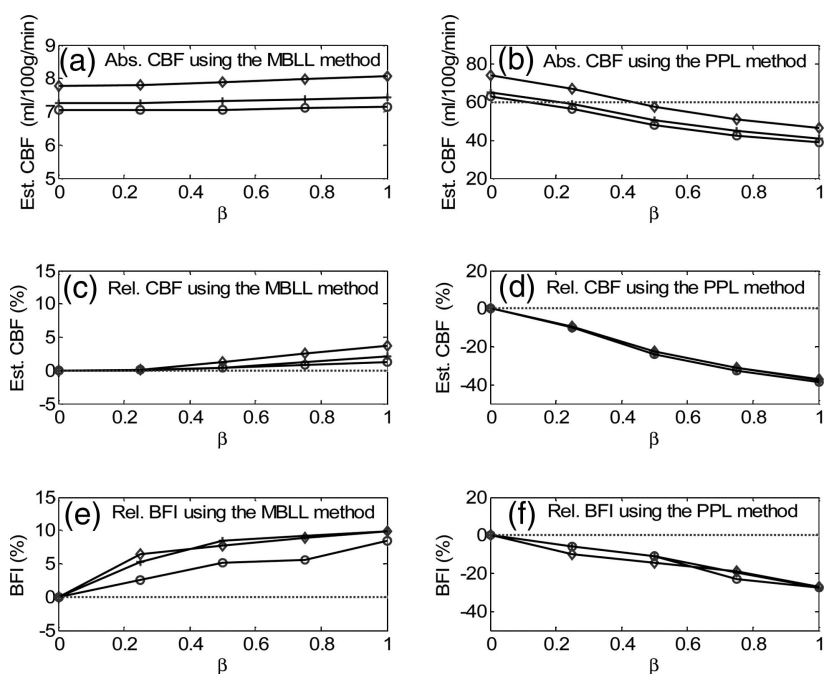

Fig. 5. Numerical Experiment 3: Both the CBF (the lower layer) and the extracerebral blood flows (the upper layer) were kept constant at 60 and $6.5 \mathrm{ml} / 100 \mathrm{~g} / \mathrm{min}$, respectively. The $\beta$ dispersion factor in the intracerebral layer varied between 0 and $1 \mathrm{~s}$ (in steps of $0.25 \mathrm{~s}$ ). (a), (c), and (e) Results obtained using the MBLL method. (b), (d), and (f) Results obtained using the PPL method. The results are presented as absolute values $(\mathrm{ml} / 100 \mathrm{~g} / \mathrm{min})$ for estimated $\mathrm{CBF}$ s in (a) and (b) and as relative values (\% change from the first value when $\beta=0 \mathrm{~s}$ ) for estimated CBFs and BFIs in (c), (d), (e), and (f). The results were calculated with thicknesses of the extracerebral layer at $8(\diamond), 10(+)$ and $12(\bigcirc) \mathrm{mm}$. The dotted line represents the ideal estimation of $\mathrm{CBF}$, which is not biased by any dispersion in the vasculature.

spacings of 45 and $50 \mathrm{~mm}$, respectively). An identity line (dotted line) was drawn, where appropriate, on each figure corresponding to the ideal CBF or BFI estimates.

A. Influence of the Extracerebral Blood Flow on Cerebral Blood Flow and Blood Flow Index Estimation

In the first two numerical experiments, we investigated the influence of the extracerebral blood flow on the $\mathrm{CBF}$ and BFI estimations. Figure 3 shows the results of Experiment 1 whereby the extracerebral blood flow was kept constant at $6.5 \mathrm{ml} / 100 \mathrm{~g} / \mathrm{min}$, and the real CBF varied between $-20 \%$ and $20 \%$ of its baseline value of $60 \mathrm{ml} / 100 \mathrm{~g} / \mathrm{min}$. Using the MBLL, it can be seen from Fig. 3(a) that all the estimated absolute CBF was between 6.5 and $8.5 \mathrm{ml} / 100 \mathrm{~g} / \mathrm{min}$, which are close to the constant extracerebral blood flow at $6.5 \mathrm{ml} / 100 \mathrm{~g} / \mathrm{min}$ showing its influence on the $\mathrm{CBF}$ estimates. The relative percentage changes in the estimated $\mathrm{CBF}$ and BFIs as shown in Figs. 3(c) and 3(e) do show some small changes as the underlying real CBF varies [e.g., for extracerebral thickness at $8 \mathrm{~mm}$, Fig. 3(c) shows that the percentage change of estimated CBF is only $\pm 5 \%$ for real $\mathrm{CBF}$ changes $\pm 20 \%]$. However, the sensitivities are therefore small. This means that the percentage changes of both $\mathrm{CBF}$ and BFI are largely underestimated. The sensitivity of those results corresponding to a thinner extracerebral layer (e.g., $8 \mathrm{~mm}$ ) is marginally higher. These results once again show that both the CBF estimates and the BFIs are dominated by the extracerebral blood flow, which was kept constant in this experiment.

Using the PPL method, the estimated absolute $\mathrm{CBF}$ is comparatively closer to the identity line as shown in Fig. 3(b). As discussed in Subsection 2.E, only the partial path lengths before the appearance of ICG were estimated and used for the whole tissue ICG time-concentration curve. Although this approach makes this method more readily applicable in practice, the errors introduced resulted in the discrepancies between the absolute CBF estimates and the identity line. It is also the case that the thicknesses of the extracerebral layer have an effect on these absolute CBF estimates, and in this example the absolute CBF estimates approach the identity line as the extracerebral layer thickness becomes larger, e.g., $12 \mathrm{~mm}$. Figure 3(d) shows the relative percentage changes of CBFs, and in all cases the estimates follow the identity line. Although not as good as its CBF counterpart, the percentage changes in BFIs still follow the identity line reasonably closely as shown in Fig. 3(f). It is also noted that similar relative percentage changes are observed when different extracerebral layer thicknesses are used.

In Numerical Experiment 2, the CBF was kept constant at $60 \mathrm{ml} / 100 \mathrm{~g} / \mathrm{min}$ while the extracerebral blood flow was varied between $-20 \%$ and $+20 \%$ of its baseline value of $6.5 \mathrm{ml} / 100 \mathrm{~g} / \mathrm{min}$. Using the MBLL method, Fig. 4(a) shows that the estimated absolute CBF varies linearly between 5 and $9 \mathrm{ml} / 100 \mathrm{~g} / \mathrm{min}$ in all cases, which again indicates the large influence of the extracerebral blood flow. Figure 4(c) further confirms the dominance of the varying extracerebral blood flow on the estimates and shows that the relative percentage changes of the $\mathrm{CBF}$ estimates closely follow the changes in the extracerebral blood flow. Figure 4(e) shows that the percentage changes in BFIs are equally affected by the varying extracerebral blood flow although in a nonlinear fashion.

Figure 4(b) shows the absolute CBF estimates using the PPL method. It can be seen that the estimates are in general insensitive to changes in the extracerebral blood flow. The discrepancies between the real $\mathrm{CBF}$ at $60 \mathrm{ml} / 100 \mathrm{~g} / \mathrm{min}$ and the estimated values depend on the thickness of the extracerebral layer. In this example, the discrepancy is smallest when the thickness is $12 \mathrm{~mm}$. The insensitivities of the $\mathrm{CBF}$ estimates and the BFI to varying extracerebral blood flow are further illustrated in Figs. 4(d) and 4(f) where the relative percentage changes are almost zero in all cases. It is also noted that similar relative percentage changes are observed when different extracerebral layer thicknesses are used.

\section{B. Influence of the Vasculature Dispersion on Cerebral Blood Flow and Blood Flow Index Estimation}

In Numerical Experiment 3, both the extracerebral blood flow and the CBF were kept constant at 6.5 and $60 \mathrm{ml} / 100 \mathrm{~g} / \mathrm{min}$, respectively, while the dispersion factor for the intracerebral layer varied between 0 and $1 \mathrm{~s}$ (in steps of $0.25 \mathrm{~s}$ ). Figures 5(a), 5(c), and 5(e) 
show the results obtained using the MBLL method. As discussed in Subsection 3.B, the $[\operatorname{ICG}(t)]_{\text {tis }}$ obtained using this method is dominated by the extracerebral blood flow, and this is confirmed by Fig. 5(a) in which the absolute CBF estimates are much smaller than the real CBF. Ideally, one would hope that the CBF estimates and the BFIs would remain constant and not be altered by the dispersion, i.e., zero relative percentage changes. The relative percentage changes of CBF estimates and BFIs shown in Figs. 5(c) and 5(e) increase by approximately 5\% and $10 \%$, respectively (for extracerebral thickness of 8 $\mathrm{mm}$ ), as the dispersion factor increases from 0 to $1 \mathrm{~s}$. These erroneous changes are in fact smaller than those obtained by the PPL method as shown in Figs. 5(d) and 5(f). However, it does not mean that the MBLL method is more robust in the presence of dispersion but that $[\operatorname{ICG}(t)]_{\text {tis }}$ calculated using the MBLL method is less sensitive to intracerebral changes. This can be better explained by referring to Fig. 2(a) in which $[\mathrm{ICG}(t)]_{\operatorname{det} 2}\left([\mathrm{ICG}(t)]_{\text {tis }}\right.$ measured by detector 2) corresponding to three dispersion factors in the intracerebral layer are plotted. It can be seen that their shapes are similar to each other, indicating that any changes in the intracerebral layer has small influence on $[\mathrm{ICG}(t)]_{\operatorname{det} 2}$.

Using the PPL method, the absolute CBF estimates are reduced by a considerable amount as the dispersion factor increases from 0 to $1 \mathrm{~s}$ as shown in Fig. 5(b). Figure 5(d) shows the relative percentage changes, which are as much as $-40 \%$ when the dispersion factor is $1 \mathrm{~s}$. The strong effect of dispersion on the $\mathrm{CBF}$ estimates can be better appreciated by referring to Fig. 2(b), which shows that $[\operatorname{ICG}(t)]_{\operatorname{det} 1 \& 2}$ becomes more dispersed and has a lower peak value as the dispersion factor is increased from 0 to 1 . These characteristics of the curves translate to a large reduction of the $\mathrm{CBF}$ estimates after deconvolution. As for the BFI in Fig. 5(f), the relative percentage changes are reduced by approximately $-25 \%$ when the dispersion factor increases from 0 to $1 \mathrm{~s}$. Since, the BFI is calculated from the initial rise of $[\operatorname{ICG}(t)]_{\operatorname{det} 1 \& 2}$ and is not influenced by the dispersion of the remaining part of the curve, it turns out that the reduction in the percentage change in BFI is smaller than those in the $\mathrm{CBF}$ estimates.

\section{Discussions and Conclusions}

The use of NIRS and ICG in assessing cerebral hemodynamic variables has been attempted by many research groups. It has been reported that the qualitative shape of the measured tissue ICG concentration time curves can reflect the health of cerebral perfusion. ${ }^{36,37}$ However, turning the tissue ICG time-concentration curves into quantitative CBF estimates in $\mathrm{ml} / 100 \mathrm{~g} / \mathrm{min}$ or BFIs is more difficult and controversial. We have shown through computer simulations that $\mathrm{CBF}$ (absolute and relative) and BFIs (relative) estimated using the MBLL method (one detector) are heavily weighted toward the extracerebral blood flow.
The PPL method (two detectors) improves the sensitivities of the $\mathrm{CBF}$ estimates (absolute and relative) and the BFIs (relative) to the underlying real $\mathrm{CBF}$ changes and reduces the influence of varying extracerebral blood flows (increasing robustness). An accurate quantification of absolute CBF in $\mathrm{ml} / 100 \mathrm{~g} / \mathrm{min}$ using this method, on the other hand, is difficult because the PPL method involves the estimation of partial path lengths using computer simulations and therefore requires accurate measurements of optical properties in each layer, which are difficult to obtain in practice. As for BFI as a relative measure, it should be noted that the initial rise of a tissue ICG concentration time curve is not linear, and subsequently the corresponding BFI does not increase linearly with the underlying real $\mathrm{CBF}$. This explains the lack of linearity of BFIs we found in Figs. 3(e), 3(f), 4(e), 4(f), $5(\mathrm{e})$, and 5(f). Our results suggest that using the $\mathrm{CBF}$ estimates obtained from the PPL method as relative measures to quantify percentage changes from a baseline value is by far the most reliable approach to assess cerebral perfusion. Different extracerebral thicknesses also do not seem to influence these percentage changes significantly as demonstrated in Figs. 3(d) and 4(d).

The problem becomes more complicated when the dispersion in the vasculature is taken into account. Figure 5 shows that the CBF estimates and the BFIs are biased in the presence of dispersion even using the PPL method. This is in fact a more fundamental problem of the dilution indicator methodology. The IRF approach cannot distinguish tracer dispersion in the feeding vessels, i.e., $[\operatorname{ICG}(t)]_{\text {art }}$ from tracer retention in the capillary bed of the field of view, i.e., $[\mathrm{ICG}(t)]_{\mathrm{int} .}{ }^{20,38}$ This limitation is particularly relevant in NIRS measurements because $[\operatorname{ICG}(t)]_{\mathrm{art}}$ is often measured at a peripheral site (e.g., nose or ear lobe), which is some distance away from the actual feeding vessels in the brain. It has been suggested that resolving this problem requires the modeling of the vasculature, which has been attempted in the MRI bolus tracking technique. ${ }^{38}$

Apart from the PPL approach, one other possible method to estimate the optical properties in the two layers separately is to use the two-layered optical model for inversion. ${ }^{15}$ This has not been done here because it is generally not valid to use the same model for forward modeling and inversion. However, in general the two-layered model can be used for inversion in experimental measurements although there may be issues concerning long computation time and robustness.

We do not expect the two-layered optical model used here to be able to accurately predict the actual optical data measured in a real human head. The two-layered model is a gross simplification in that the complicated layered structure of a head is simplified to two flat layers corresponding to the extracerebral and intracerebral layers. Also, the cerebrospinal fluid has been ignored. Okada and Delpy ${ }^{25}$ showed that the presence of low-scattering cerebrospinal fluid increases the partial path length in the brain and hence 
the actual optical measurements on the surface of the head may have a higher sensitivity to the real CBF than our results shown. In any case, the relatively effective method we found here, namely, the PPL method, involves the weighted subtraction of $\mu_{a}$ as measured by two detectors. Any discrepancies attributable to the use of an inaccurate optical model apply equally to both $\mu_{a}$ and are substantially canceled out in the subtraction. We therefore expect the results obtained from the PPL method using the proposed modeling technique to be sufficiently valid. In a recent experimental study in which the CBFs of ten adult volunteers were increased (as assessed by transcranial Doppler) by the administration of acetazolamide, we found that the velocity of the middle cerebral artery as measured by transcranial Doppler was increased by $49 \pm 21 \%$. However, the BFIs calculated using the MBLL method were unable to detect a statistically significant increase of the underlying CBF. ${ }^{9}$ We are currently investigating the use of the PPL method in this set of data.

When different baseline physiological or optical parameters (e.g., water content or $\mu_{a}^{b k}$ ) are used, the absolute CBF and BFIs obtained will also differ because the general distribution of light would have been changed as a result. We carried out a test (results not shown here) in which the extracerebral and intracerebral $\mu_{a}^{b k}$ were reduced to half of the values shown in Table 1. Unsurprisingly, we found different values for the absolute $\mathrm{CBF}$ and BFIs. However, the central messages stated here still applied, i.e., (1) using the MBLL method, the absolute CBF was significantly underestimated, (2) the PPL method improved the accuracy and robustness, (3) the CBF estimates were best used as relative measures, and (4) the dispersion of the AIF affected the CBF estimates.

The authors thank Hamamatsu Photonics KK, University College London and University College Hospital Trustees, the Interdisciplinary Research Collaboration of the Engineering and Physical Sciences Research Council and Medical Research Council (grant 075608) for their support of this work. We also thank Alwin Kienle for his help with the implementation of the two-layer analytical model.

\section{References}

1. T. Durduran, G. Yu, M. G. Burnett, J. A. Detre, J. H. Greenberg, J. Wang, C. Zhou, and A. G. Yodh, "Diffuse optical measurement of blood flow, blood oxygenation, and metabolism in a human brain during sensorimotor cortex activation," Opt. Lett. 29, 1766-1768 (2004).

2. C. E. Elwell, M. Cope, A. D. Edwards, J. S. Wyatt, D. T. Delpy, and E. O. R. Reynolds, "Quantification of adult cerebral hemodynamics by near-infrared spectroscopy,” J. Appl. Physiol. 77, 2753-2760 (1994).

3. J. Patel, K. Marks, I. Roberts, D. Azzopardi, and A. D. Edwards, "Measurement of cerebral blood flow in newborn infants using near-infrared spectroscopy with indocyanine green," Pediatr. Res. 43, 34-39 (1998).

4. R. Springett, Y. Sakata, and D. T. Delpy, "Precise measurement of cerebral blood flow in newborn piglets from the bolus passage of indocyanine green," Phys. Med. Biol. 46, 2209-2225 (2001).

5. C. R. J. C. Newton, D. A. Wilson, E. Gunnoe, B. Wagner, M. Cope, and R. J. Traystman, "Measurement of cerebral blood flow in dogs with near-infrared spectroscopy in the reflectance mode is invalid," J. Cereb. Blood Flow Metab. 17, 695703 (1997).

6. W. M. Kuebler, A. Sckell, O. Habler, M. Kleen, G. E. H. Kuhnle, M. Welte, K. Messmer, and A. E. Goetz, "Noninvasive measurement of regional cerebral blood flow by near-infrared spectroscopy and indocyanine green," J. Cereb. Blood Flow Metab. 18, 445-456 (1998).

7. F. Gora, S. Shinde, C. E. Elwell, J. C. Goldstone, M. Cope, D. T. Delpy, and M. Smith, "Measurement of cerebral blood flow in adults using near-infrared spectroscopy and indocyanine green,” J. Neurosurg. Anesthesiol. 14, 218-222 (2002).

8. B. P. Wagner, S. Gertsch, R. A. Ammann, and J. Pfenninger, "Reproducibility of the blood flow index as noninvasive, bedside estimation of cerebral blood flow," Intensive Care Med. 29, 196-200 (2003).

9. I. Tachtsidis, T. S. Leung, M. Tisdall, D. T. Delpy, M. Smith, and C. E. Elwell, "Cerebral blood flow assessment with indocyanine green bolus transit detection by near-infrared spectroscopy before and after acetazolamide provocation in humans," in Biomedical Optics 2006 Technical Digest (Optical Society of America, 2006), ME67.

10. K. Zierler, "Equations for measuring blood flow by external monitoring of radioisotopes," Circ. Res. 16, 309-321 (1965).

11. G. T. Gobbel and J. R. Fike, "A deconvolution method for evaluating indicator-dilution curves," Phys. Med. Biol. 39, 1833-1854 (1994).

12. D. W. Brown, P. Picot, J. Gharavi, R. Springett, D. T. Delpy, R. Menon, V. Han, and T. Y. Lee, "Quantitative NIRS measurement of cerebral hemodynamics in newborn piglets," Pediatr. Res. 51, 564-570 (2002).

13. E. Keller, A. Nadler, H. Alkadhi, S. S. Kollias, Y. Yonekawa, and P. Niederer, "Noninvasive measurement of regional cerebral blood flow and regional cerebral blood volume by nearinfrared spectroscopy and indocyanine green dye dilution," Neuroimage 20, 828-839 (2003).

14. R. D. Rothoerl, K. M. Schebesch, R. Faltermeier, C. Woertgen, and A. Brawanski, "Lack of correlation between Xenon ${ }^{133}$ and near-infrared spectroscopy/indocyanine green rCBF measurements," Neurol. Res. 25, 528-532 (2003).

15. A. Kienle, M. S. Patterson, N. Dognitz, R. Bays, G. Wagnieres, and $H$. van den Bergh, "Noninvasive determination of the optical properties of two-layered turbid media," Appl. Opt. 37, 779-791 (1998).

16. P. Meier and K. L. Zierler, "On the theory of the indicatordilution method for measurement of blood flow and volume," J. Appl. Physiol. 6, 731-743 (1954).

17. L. Friberg, J. Kastrup, M. Hansen, and J. Bulow, "Cerebral effects of scalp cooling and extracerebral contribution to calculated blood flow values using the intravenous 133Xe technique," Scand. J. Clin. Lab. Invest. 46, 375-379 (1986).

18. R. M. P. Doornbos, R. Lang, M. C. Aalders, F. W. Cross, and H. J. C. M. Sterenborg, "The determination of in vivo human tissue optical properties and absolute chromophore concentrations using spatially resolved steady-state diffuse reflectance spectroscopy," Phys. Med. Biol. 44, 967-981 (1999).

19. J. S. Wyatt, M. Cope, D. T. Delpy, C. E. Richardson, A. D. Edwards, S. Wray, and E. O. Reynolds, "Quantitation of cerebral blood volume in human infants by near-infrared spectroscopy," J. Appl. Physiol. 68, 1086-1091 (1990).

20. F. Calamante, D. G. Gadian, and A. Connelly, "Delay and dispersion effects in dynamic susceptibility contrast MRI: simulations using singular value decomposition," Magn. Reson. Med. 22, 466-473 (2000). 
21. R. W. Stow and P. S. Hetzel, "An empirical formula for indicator-dilution curves as obtained in human beings," J. Appl. Physiol. 7, 161-167 (1954).

22. K. Murase, M. Shinohara, and Y. Yamazaki, "Accuracy of deconvolution analysis based on singular value decomposition for quantification of cerebral blood flow using dynamic susceptibility contrast-enhanced magnetic resonance imaging," Phys. Med. Biol. 46, 3146-3159 (2001).

23. M. Kohl-Bareis, H. Obrig, J. Steinbrink, J. Malak, K. Uludag, and A. Villringer, "Noninvasive monitoring of cerebral blood flow by a dye bolus method: separation of brain from skin and skull signals," J. Biomed. Opt. 7, 464-470 (2002).

24. P. Mansfield, "Imaging by nuclear magnetic resonance," J. Phys. E 21, 18-30 (1988).

25. E. Okada and D. T. Delpy, "Near-infrared light propagation in an adult head model. I. Modeling of low-level scattering in the cerebrospinal fluid layer," Appl. Opt. 42, 2906-2914 (2003).

26. M. Essenpreis, C. E. Elwell, P. van der Zee, S. R. Arridge, and D. T. Delpy, "Spectral dependence of temporal point spread functions in human tissues," Appl. Opt. 32, 418-425 (1993).

27. D. M. Hueber, M. A. Franceschini, H. Y. Ma, Q. Zhang, J. R. Ballesteros, S. Fantini, D. Wallace, V. Ntziachristos, and B. Chance, "Noninvasive and quantitative near-infrared haemoglobin spectrometry in the piglet brain during hypoxic stress, using a frequency-domain multidistance instrument," Phys. Med. Biol. 46, 41-62 (2001).

28. Y. Hoshi, M. Shimada, C. Sato, and Y. Iguchi, "Reevaluation of near-infrared light propagation in the adult human head: implications for functional near-infrared spectroscopy," J. Biomed. Opt. 10, 064032 (2005).

29. S. J. Matcher and C. E. Cooper, "Absolute quantification of deoxyhaemoglobin concentration in tissue near-infrared spectroscopy,” Phys. Med. Biol. 39, 1295-1312 (1994).

30. P. van der Zee, M. Essenpreis, and D. T. Delpy, "Optical properties of brain,” Proc. SPIE 1888, 454-465 (1993).
31. S. J. Matcher, M. Cope, and D. T. Delpy, "In vivo measurements of the wavelength dependence of tissue-scattering coefficients between 760 and $900 \mathrm{~nm}$ measured with time-resolved spectroscopy," Appl. Opt. 36, 386-396 (1997).

32. A. Duncan, J. H. Meek, M. Clemence, C. E. Elwell, P. Fallon, L. Tyszczuk, M. Cope, and D. T. Delpy, "Measurement of cranial optical-path length as a function of age using phaseresolved near-infrared spectroscopy," Pediatr. Res. 39, 889894 (1996)

33. M. Hiraoka, F. Firbank, M. Essenpreis, M. Cope, S. R. Arridge, P. van der Zee, and D. T. Delpy, "A Monte Carlo investigation of optical path length in inhomogeneous tissue and its application to near-infrared spectroscopy," Phys. Med. Biol. 38, 1859-1876 (1993).

34. W. H. Press, S. A. Teukolsky, W. T. Vetterling, and B. P. Flannery, Numerical Recipes in C, 2nd ed. (Cambridge U. Press, 1992).

35. L. Ostergaard, R. M. Weisskoff, D. A. Chesler, C. Gyldensted, and B. R. Rosen, "High-resolution measurement of cerebral blood flow using intravascular tracer bolus passages. Part I: Mathematical approach and statistical analysis," Magn. Reson. Med. 36, 715-725 (1996).

36. E. Keller, M. Wolf, M. Martin, and Y. Yonekawa, "Estimation of cerebral oxygenation and hemodynamics in cerebral vasospasm using indocyanine green dye dilution and near-infrared spectroscopy," J. Neurosurg. Anesthesiol. 13, 43-48 (2001).

37. A. Liebert, H. Wabnitz, J. Steinbrink, M. Moller, R. Macdonald, H. Rinneberg, A. Villringer, and H. Obrig, "Bedside assessment of cerebral perfusion in stroke patients based on optical monitoring of a dye bolus by time-resolved diffuse reflectance," Neuroimage 24, 426-435 (2005).

38. L. Ostergaard, D. A. Chesler, R. M. Weisskoff, A. G. Sorensen, and B. R. Rosen, "Modeling cerebral blood flow and flow heterogeneity from magnetic resonance residue data," J. Cereb. Blood Flow Metab. 19, 690-699 (1999). 